\title{
The Impact of Incorporating Wind Energy in the Electric Grid
}

\author{
B. A. Carreras \\ Depart. Fisica, Universidad \\ Carlos III, Madrid, Spain \\ bacarreras@gmail.com
}

\author{
J. M. Reynolds Barredo \\ Depart. Fisica, Universidad \\ Carlos III, Madrid, Spain \\ jmrb2002@gmail.com
}

\author{
D. E. Newman \\ Physics Dept. \\ University of Alaska Fairbanks \\ denewman@alaska.edu
}

\begin{abstract}
In this paper we investigate the impact of increasing the penetration of wind generation with real variability on the risk to, and robustness of, the power transmission grid using a dynamic model of the power transmission system (OPA). There are three timescales of variability discussed but this paper will focus on the impact of two. It is found that with different fractions and distributions of wind generation and central generation, varied dynamics and risk are possible. One important parameter is the fraction of the total power demand supplied by the wind generation. It is found that the risk has a minimum in fraction of wind power supplied, after which the risk increased as the wind power penetration increases. In the same networks, decreasing the number of central generators without decreasing their power supplied in general increases the risk after a critical minimum number of generators is reached.
\end{abstract}

\section{Introduction}

Around the world there is an increasing role, often mandated, of renewable energy sources in electricity production [1]. These sources include wind, solar and small scale hydro among others. This increased utilization of new energy sources opens many issues regarding the impact of this increased penetration on the reliability of the power grid. One important factor in many of these new sources is the introduction of the question of variability of the source and its impact on the operation of the grid. This issue of variability is also coupled to the question of energy storage [2] to continuously meet power demands that opens a whole new field of research [3].
The variability of these energy sources appears at many different time scales. Three are of particular interest, short, up to minutes or hours, medium, days to months and long, months to years. On the short time scale, a key controlling variable is the ramp rates of generators. Hydro and gas have high ramp rates and are currently used to compensate for wind and sun energy fluctuations during an hour. On the medium time scale the generation and grid has to adapt to the daily and weekly fluctuations which can be very challenging for storage and is often difficult to accurately predict. Finally, on a longer time scale, up to seasonal variations of the renewable energy sources the variability is fairly repeatable and therefore fairly easy to predict. However, on this longest time scale, when the seasonal variation is large, storage becomes impractical. For the shortest to medium time scales there is more interest in having the renewable sources coupled to a storage system that must be effective and economical.

In this paper, we focus on the daily and longer time scales with the goal being to understand the impact on grid reliability and operation with varying degrees of penetration of these variable energy sources when coupled to a storage system that is effective up to the medium time scale. The point being that the transmission grid under these conditions of high variability and therefore constantly changing dispatch is under a higher degree of stress which may affect its performance.

In order to use relatively reliable quantitative models of the energy source, we use wind and wind generation models for these initial studies that will be later extended to multiple types of renewable energy sources.

The incorporation of renewable sources of energy to the electric grid although being very desirable is not a simple process by itself. The variability of these energy sources makes it very difficult to simply substitute the fossil fuel plants with wind plants. Here, we discuss a process through which we try to connect these plants to an existing grid in an efficient manner. 
This is not a fully realistic process, but helps in understanding the problems that would be faced in doing so. In time and by improving many of the problems we face, it could lead to an efficient method for planning this process.

The method that we use is based on simulations of the electric grid operation with the OPA code [4-6] and going though a sequence of steps in order to achieve our goal. The main steps are:

1) Optimization of the power flow out of the wind power plant in a way that we minimize the storage needs on the medium time scale.

2) Add to the basic network a number of possible wind plants on the periphery of the net and attach them to nodes more likely to have blackouts. In this work we add the wind plants to the periphery, future work will look at optimizing the location of the additions across the entire grid.

3) Vary the power produced by the wind plants in order to find the most effective size of the wind plants for the operation of the network.

4) Reduce the number of fossil fuel plants maintaining the reliability of the overall grid and eliminate wind plants that are not needed.

The idea is then by using this process we can effectively switch out a maximum number of fossil fuel plants with wind plants without increasing the risk in the operation of the power system. We do that using several different starting configurations and several approaches. These fours steps are discussed in the next four Sections. In Section 6, we compare the final results and give the conclusions of this study.

\section{Optimization of the power flow out of the wind power plant}

The first step in this work is to make a model for the wind electricity production that can be incorporated in the OPA code in order to evaluate the dynamics of the power transmission model with wind production. In order to build this model, we first have analyzed wind data from the north of California [7]. These data from different locations present similar characteristics. There is an annual slow variation, with a maxima in the summer months, and a high level of fluctuations on top of these mean evolution. An example is shown in Fig. 1 .

In Fig. 1, we have plotted the cube of the velocity because the power production of the windmills is proportional to this quantity. We show only the daily variation because in this first study we will limit ourselves to looking at the generation in daily steps. We also show a fit to the data based on a sinusoidal function that describes the slow variation of the wind power during the year.
An important issue related to the power production is the issue of energy storage. Many methods of energy storage are being developed just now [2]. Here we do not discuss the various storage approaches, their feasibility, costs or pro and cons, we just assume that an efficient one exists that can supply the needed storage up to the daily variability time scale. We can calculate the energy storage needed in order to be able to guarantee an average power supply in the presence of the highly variable wind power production. First we need to evaluate what the daily power flow is that can be delivered to the customers in order to maximize efficiency and minimize the cost of storage. If $\mathrm{P}(\mathrm{t})$ is the wind power produced every day and $P_{F}(t)$ is the power flow out of the plant, we can estimate the energy storage needed to maintain the power flow by calculating:

$$
R(t)=\int_{0}^{f}\left(P\left(t^{\prime}\right)-P_{F}\left(t^{\prime}\right)\right) d t^{\prime}
$$

The maximum value of $\mathrm{R}$ gives us the storage needed. We have to calculate the power flow out $P_{F}(t)$ by minimizing the maximum value of $\mathrm{R}$ with the condition $\mathrm{R}>0$.

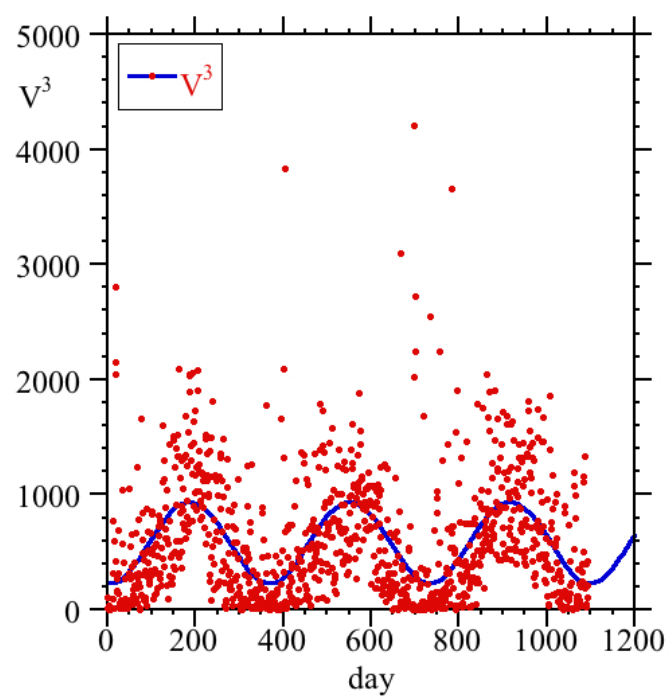

Fig. 1. Daily variation of the cube of the wind velocity and a fit showing the slow yearly variation

Because of the large annual variation and the desire to use this technique for the daily variability we separate the power flow out $\mathrm{P}_{\mathrm{F}}(\mathrm{t})$ by month in such a way that the company may contract a fixed power production each month. Then we have developed a simple optimization algorithm based on a Monte Carlo 
approach that allows the calculation of the power flow that should be used to minimize the storage and while keeping the condition $\mathrm{R}>0$. We have applied this method to several sets of wind data. An example of the resulting power flow out and wind power are shown in Fig. $2 \mathrm{a}$ and storage needed is shown in Fig. $2 \mathrm{~b}$.

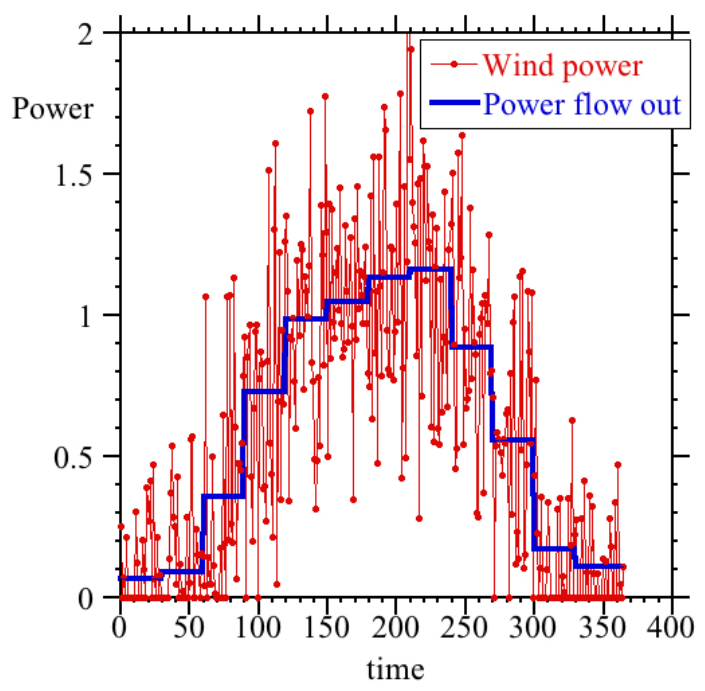

Fig. 2a. Power flow model for a simple example.

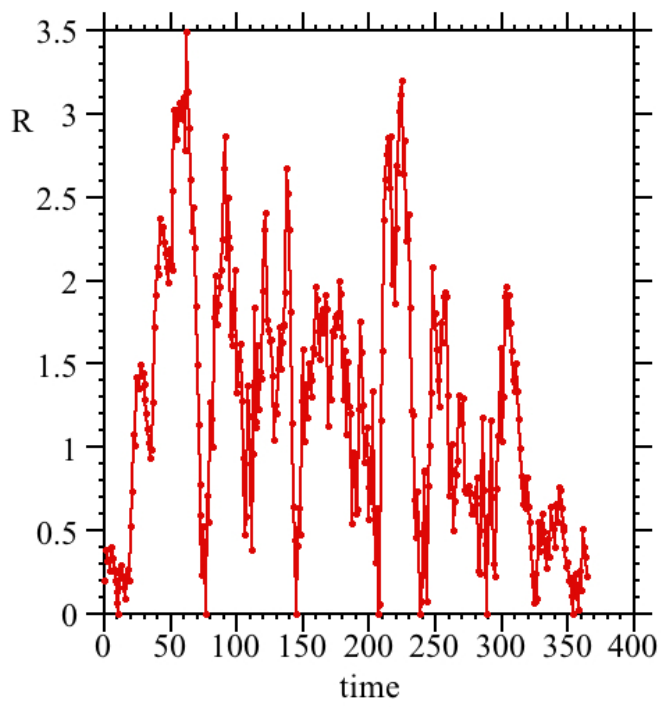

Fig. $2 b$. Storage needs for the same simple example.

For the power flow out of the plant shown in Fig. 2, the PDF of the fluctuations of the wind power produced around the mean flow delivered is shown in
Fig.3. This PDF is also approximately described by a gaussian distribution.

Using this information, we construct a model to be use in the OPA code. In this model, the power flow out is given by the function $P_{F}(t)$ we have just calculated. The power produce by the plant, $\mathrm{P}(\mathrm{t})$, is equal to $P_{F}(t)$ plus a daily random value given by the Gaussian distribution in Fig. 3. If $\mathrm{P}(\mathrm{t})$ is greater than $P_{F}(t)$, the excess power is accumulated in the storage, if it is smaller power is taken out from the storage. In case there is not enough power stored, the power delivered is less than $\mathrm{P}_{\mathrm{F}}(\mathrm{t})$. We also set up two generation limits. The days in which the wind power production goes above a given limit, the power production is set to zero, because this very high production implies very strong winds and windmills cannot operate in this condition. Also if the production goes below a second limit, the production is set to zero.

It is important to note that we are using this model to examine the impact of these various types of variability and control of the the variability on the reliability of the power transmission grid. Therefore, the underlying details of the wind generation model and storage are not important as long as they capture the basic time series characteristics. We are also assuming that each wind generation facility has its own power storage co-located with the generation. This will of course not always be the case and the impact of generalizations of the storage locations will be investigated in future work.

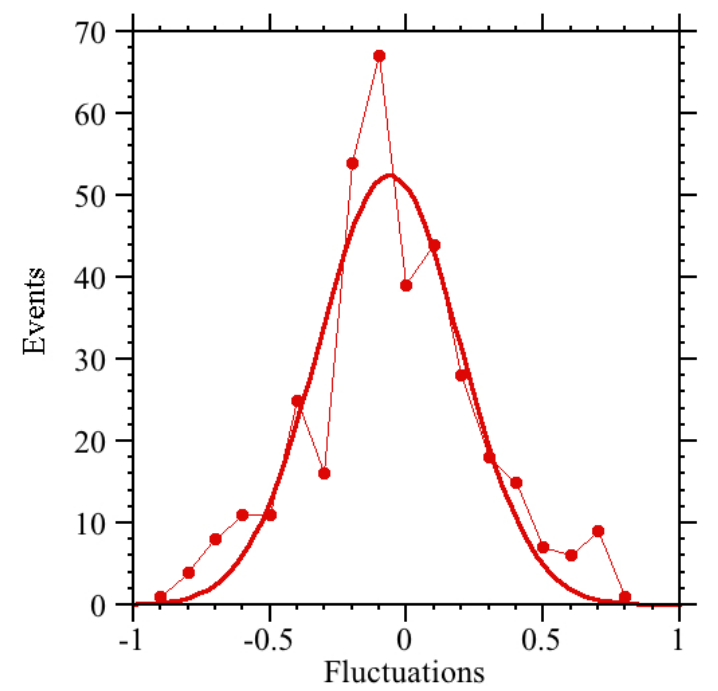

Fig. 3. PDF of the daily wind fluctuations for the model in Fig.2 and a gaussian fit

To quantify the proportion of wind power contributed to the grid we use the time average of the ratio of the sum of generation limits of the wind plants, $P_{W}$ divided by the total power demand, $P_{D}$. 
For the power dispatch, we use a cost for the wind generation, which is half of the cost of the other generation plants. In this way, the wind generation is dispatched first. Using smaller values of the cost, like $5 \%$ of the other costs, did not make any difference for the cases considered here.

\section{Basic network with a number of possible wind plants}

To study the various ways to incorporate wind plants into existing power transmission networks, we start by using small artificial networks [8], for instance the 200 and 400 node artificial networks with network characteristics similar to real power transmission grids. The 200 node network has 37 generator nodes and the 400 node network 59. To these artificial networks, we have added generator nodes on the periphery corresponding to the wind power plants. We then use different criteria to add these nodes. One method was adding them randomly, another was to add to the nodes that are more likely to have blackouts. This second approach seems to work the best. These criteria are not realistic, however since we are using artificial networks, we do not have geographical locations to proceed in a more realistic way. When we start to study real networks, we will modify the criteria to adapt it to the geography of the location. Once we have added the nodes corresponding to the wind power generators, we run the OPA code with this new network. In this way, we know which lines have to be upgraded, and by how much, as a consequence of the addition of new generators. This then completes the construction of these new networks

In Fig. 4a, we show the 200 node network with 40 and in Fig. 4b with 60 added wind nodes. The wind nodes are colored.

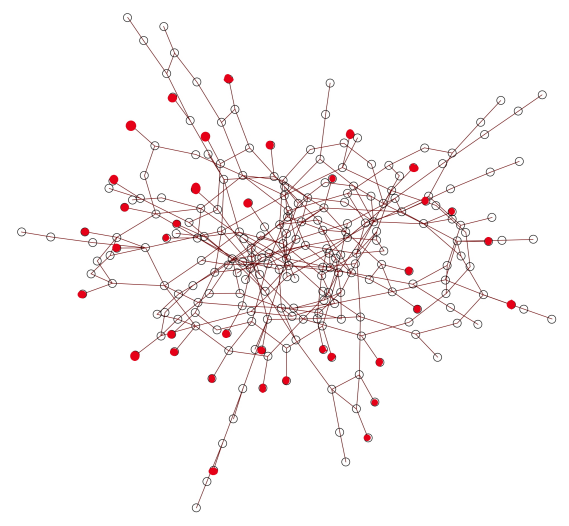

Fig. 4a The 200 node network with 40 wind nodes added

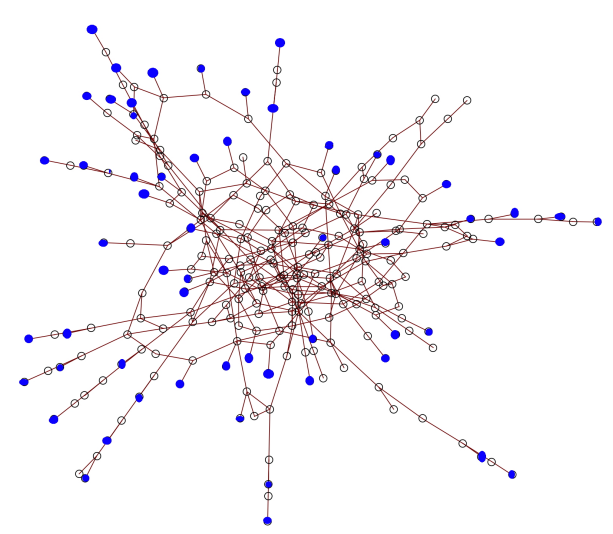

Fig. 4b. The 200 node network with 60 wind nodes added

In these two networks, we have separated the wind nodes into several groups according their location and assumed that in each group the wind properties are similar and therefore the variability of the wind in each group is the same but the variability across the groups is not correlated. This is important because the more regions there are, the smaller the overall variability in the total wind power being supplied to the system. However at the same time this increases the power being transferred between the regions which can add stress to the system. An example of the 200 node network with three groups of wind generation is shown in Fig. 5, the nodes of each group are colored differently. We have followed the same process with an initial artificial network with 400 nodes. When we follow the time evolution of these networks, we can follow the usage of the storage. Combining wind plants with different variability may reduce the storage needs which can then be taken into account. We then use all these networks in the calculations of OPA which will be discussed in the next sections.

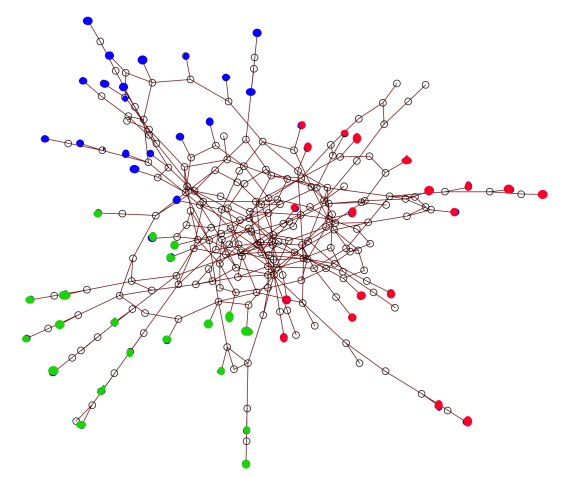

Fig. 5. The 200 node network with the added 60 wind nodes divided in three groups 


\section{Optimal power generation of the wind power plants}

Next step is to incorporate the wind power plant model and the corresponding energy storage discussed in Section 2 in OPA. On a given day, if the power production is higher than the expected power flow out, the difference is added as energy in the storage. If the production is lower than the expected power flow, we get power from the storage to serve as close as possible the corresponding power flow. With this tool we can investigate the the impact of changing both the penetration of the wind power and the distribution of the wind and central generation.

In what follows we use $<\mathrm{P}_{\mathrm{W}} / \mathrm{P}_{\mathrm{D}}>$ as a measure of the generation capacity of the wind plants, that is the average ratio of the power wind production to the total demand. Because of the annual and daily variability of the wind, the actual ratio $\mathrm{P}_{\mathrm{W}} / \mathrm{P}_{\mathrm{D}}$ varies the day to day.

As an example, in Fig. 6 for the 200 node network with 60 wind nodes, we show the actual distribution of the values of $\mathrm{P}_{\mathrm{W}} / \mathrm{P}_{\mathrm{D}}$ for two different values of $<\mathrm{P}_{\mathrm{W}} /$ $\mathrm{P}_{\mathrm{D}}>$. Since these distributions are not gaussian, $<\mathrm{P}_{\mathrm{W}} /$ $\mathrm{P}_{\mathrm{D}}>$ may not be the best measure of the efficiency of the system and better parameters will be investigated in the future. We can see that for the case with $<\mathrm{P}_{\mathrm{W}} / \mathrm{P}_{\mathrm{D}}>=$ 0.37 , on most of the days the power consumed is about 0.42 of the total demand, while for the case $<\mathrm{P}_{\mathrm{W}} / \mathrm{P}_{\mathrm{D}}>=$ 0.45 , that is about 0.5 of the total demand. We can also see in Fig. 6 that the range of values for which the wind electricity production contributes significantly to the grid is broad and the averaged value of $\mathrm{P}_{\mathrm{W}} / \mathrm{P}_{\mathrm{D}}$ may be a bit pessimistic.

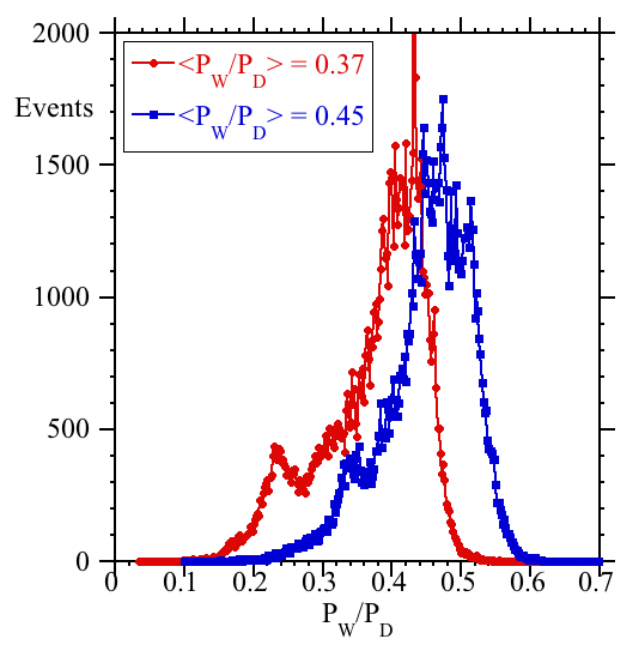

Fig. 6. PDF of the values of $P_{w} / P_{D}$ for two different values of $\left\langle\mathrm{P}_{\mathrm{w}} / \mathrm{PD}_{\mathrm{D}}\right\rangle$.
In this version of the OPA model with the wind plants, due to the daily variability of the wind, there are days with practically no wind energy production. On those days all power must be supplied by the storage or in cases where the storage can not supply the extra demand, other power plants. As we increase the proportion of wind production in the grid, the operation of the grid starts to become less reliable. Above a given value of $\left\langle\mathrm{P}_{\mathrm{W}} / \mathrm{P}_{\mathrm{D}}\right\rangle$ there is a sudden increase in the size of the blackouts and the system becomes unreliable. The best way to see that is by calculating a risk metric. We define the risk as the probability of a blackout times the cost [9], we then integrate the risk over all possible blackouts to define the risk metric. This is then normalized to the risk of the case without wind power added so 1 is the same risk as a no wind power case (all power supplied by the central generation, less then 1 means the grid has become less risky (more reliable) and greater then 1 means more risky or less reliable. We call this the normalized risk and will use it as our primary metric.

To start with we briefly look at the impact of the added "optimal" storage calculated in in section 2 on the risk. Figure 7 shows the normalized risk as a function of $<\mathrm{P}_{\mathrm{W}} / \mathrm{P}_{\mathrm{D}}>$ for 3 cases. One case (red) with the no storage, another case (green) has the optimal storage added and the final case has the daily variability (the noise) turned off (blue). These results show a few important things. First, there is an improvement in the grid for all three cases for small penetration values. Next, in the case with no storage the system reliability gets much worse quickly with the curve turning over sooner and rising rapidly. Third, the case with storage remains reliable up to higher values of wind power fraction but also turns over and starts to rise much more quickly than the case with no variability. This is at least partly because the storage is not perfect so there are times in which the storage is not able to meet the demands of the daily variability necessitating a switch to the central generation which causes a large short term stress on the grid, increasing the probability of large failures. Finally, even the case with no short term variability (the no noise case) turns up with the risk increasing as the wind power penetration increases. This is due to the slow long term (seasonal) variation of the power supplied by the wind generators. This causes an annual stress to the system and is behind much of the results that follow. All the rest of the results will include the storage and variability. 


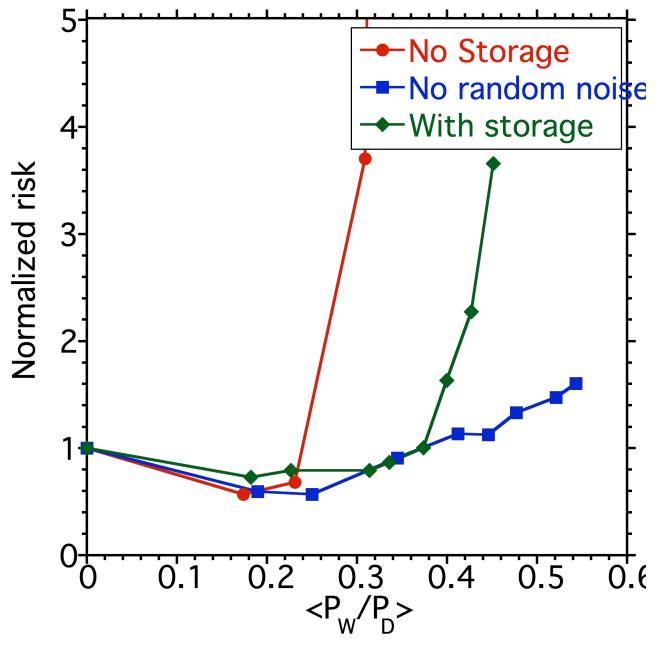

Fig. 7. Normalized blackout risk as a function of $\left\langle\mathrm{Pw}_{\mathrm{w}} / \mathrm{P}_{\mathrm{D}}\right\rangle$ for the 200 node plus 60 wind nodes network for No Storage, Storage and No daily variability (no noise) cases.

Fig. 8 shows the normalized risk for the 200 node network with different 60 added wind plants like the green curve in figure 7 but extended. It is important to note that both the frequency and size of blackouts decreases when a small amount of wind power is added to the grid. The risk as a function of $\left.<\mathrm{P}_{\mathrm{W}} / \mathrm{P}_{\mathrm{D}}\right\rangle$ then has a minimum value and starts increasing sharply. This improvement is logical because we have increased the distribution of the generation without decreasing yet the conventional generation.

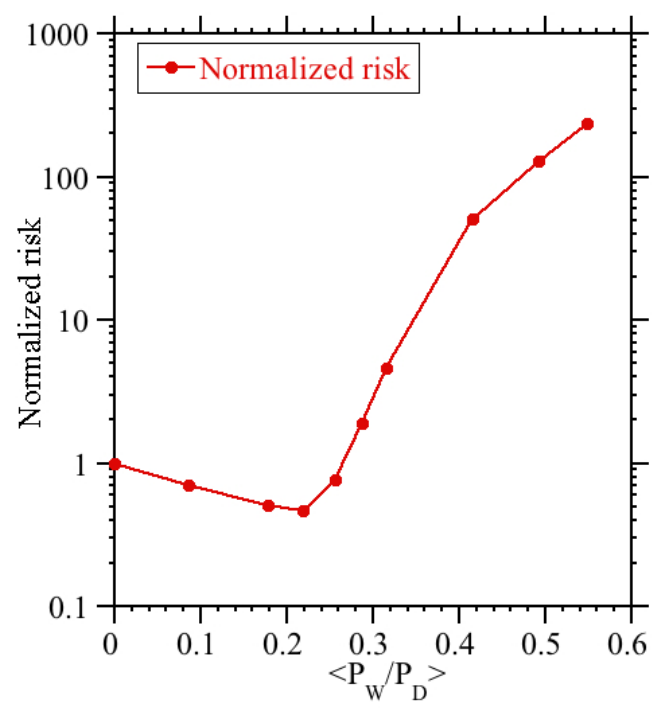

Fig. 8. Normalized blackout risk as a function of $\left\langle\mathrm{P}_{w} / \mathrm{P}_{\mathrm{D}}\right\rangle$ for the 200 node plus 60 wind nodes network.
The improvement on operation of the network is seen not only on the decrease of the frequency but in the whole reliability of the system. But once the fraction exceeds a certain value the system rapidly becomes much worse reaching a normalized risk of 100 (that is 100 times worse the the no wind base case) with just under $50 \%$ average wind fraction.

The impact also depends on how many different regions there are with the wind production synchronized in the region but not across regions. With a larger number of regions the system works better for higher wind fractions, as shown in Fig. 9. This case has 200 nodes with 60 wind plants and the region in with the rise in risk becomes sharp is moved from a fraction or $\sim 0.3$ to $\sim 0.45$ a large change. This makes sense because the different variations in the different regions partially cancel each other out. However, only the short time scale variability is canceled out meaning that there is a maximum possible improvement which would look like the no variability case in figure 7 . Another way of looking at this would be to look at the ratio of a unit of normalized risk to a unit of wind penetration. We do not expect the minimum to change substantially because the after the minimum, the increase of the risk is very fast relative to the increase in the power but will investigate this in future work.

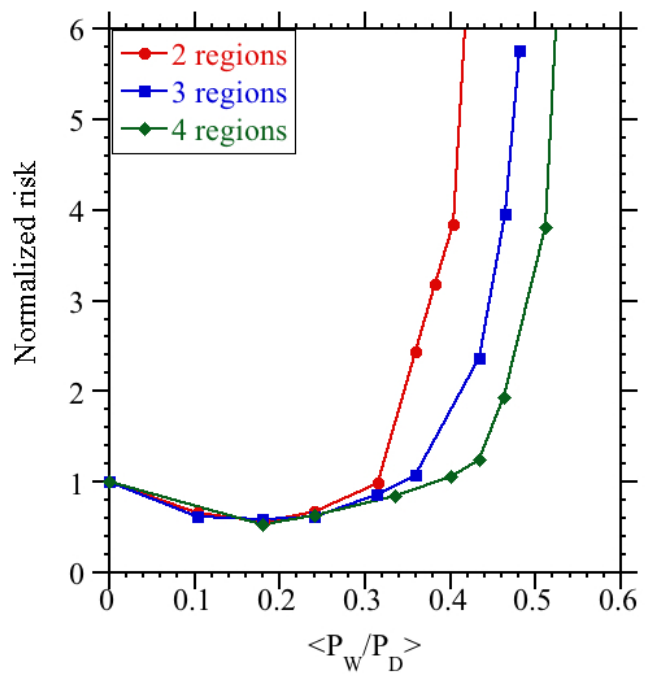

Fig. 9. Normalized blackout risk as a function of $\left\langle\mathrm{P}_{w} / \mathrm{P}_{D}\right\rangle$ for the 200 node plus 60 wind nodes network and increasing the number of regions of synchronized generation

Similar results are obtained with the 400 node network with 60 wind plants added. This can be seen in Fig. 10. 


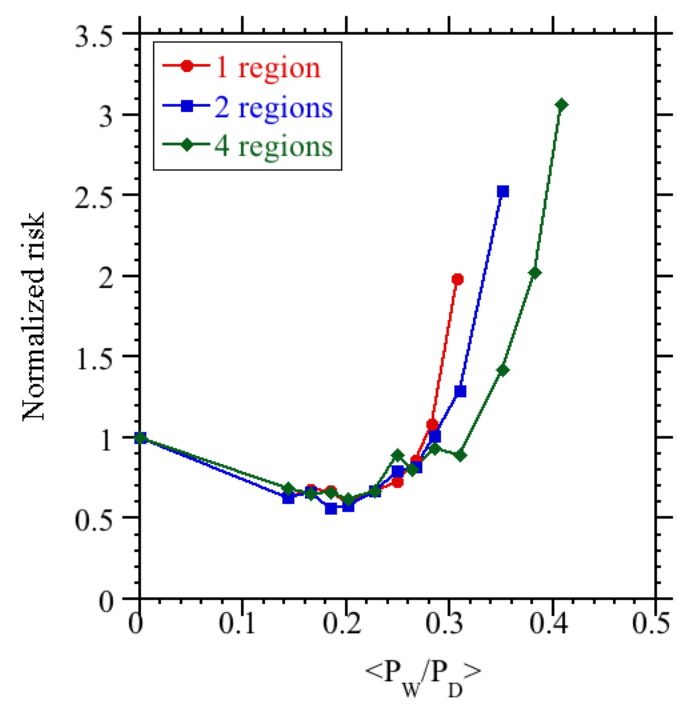

Fig. 10. Normalized blackout risk as a function of $\left\langle\mathrm{P}_{w} / \mathrm{P}_{D}\right\rangle$ for the $\mathbf{4 0 0}$ node plus 60 wind nodes network and increasing the number of regions of synchronized generation.

From these results we see that an optimal value of $<\mathrm{P}_{\mathrm{W}} / \mathrm{P}_{\mathrm{D}}>$ exists for each network which maximizes the use of the wind power without increasing the risk of blackouts. This optimal value of $\left\langle\mathrm{P}_{\mathrm{W}} / \mathrm{P}_{\mathrm{D}}>\right.$ is directly related to the power produced by each of the wind plants. This way we can determine the most efficient size of the wind power plant. Once we have that determined we can move to the next step reducing the number of central generation plants, fossil fuel plants.

\section{Reducing the number of fossil fuel plants while maintaining the reliability of the overall grid.}

To decide which power plants can be closed down (still keeping the total power fraction supplied by the conventional plants the same), we use two approaches based on the results the OPA model. For the first approach, we look for the conventional power plants that are used less over a length of time when the power is dispatched and we close a few of the least used generators and iterate the calculation to again remove the least used generators. For the second approach we use a Monte Carlo like approach and we close random sets of power plants and using the risk results from OPA evaluate which one is the best choice. For each case, the evaluation of the risk is made for a fixed system configuration, namely the number and fraction of power from the conventional generators vs the wind generators is held constant as the system runs to produce the data for the risk calculation.
For the first approach, we iterate the process of closing generators several times till the risk starts to increase sharply. We have applied this method to the 200 and 400 node networks with 60 added wind nodes. We also repeated the calculation reducing the number of wind nodes. In Fig. 11a, we show how the normalized risk of blackouts changes when we remove conventional generators for the 200 node network while in Fig. 11b the risk is shown for $11 \mathrm{~b}$. Note, the normalization of the risk is to the no reduction of conventional plants case.

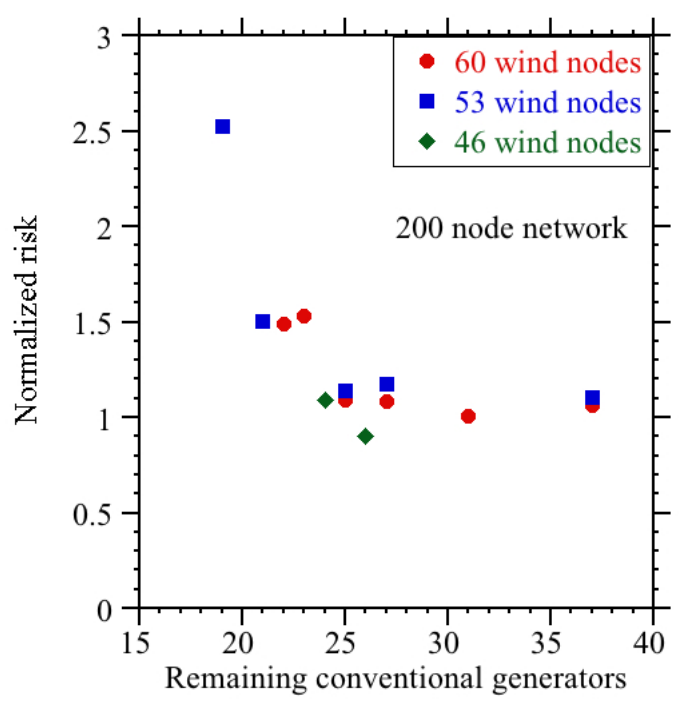

Fig. 11a. Risk as a function of the number of conventional generators for the 200 node networks.

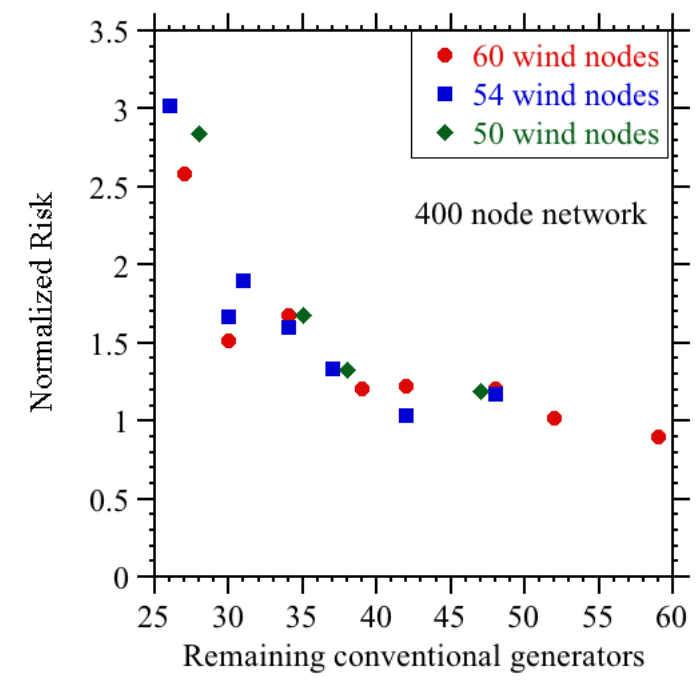

Fig. 11b. Risk as a function of the number of conventional generators for the $\mathbf{4 0 0}$ node networks. 
We can see that, for the 200 node network, we can reduce the number of conventional generator from 37 to 25 keeping the normalized risk about 1 . If we try to reduced the number further the risk of blackouts increases. For the 400 node network, we can reduce the number of conventional generators from 59 to about 40 keeping the normalized risk close to 1 before the risk starts to increase.

The second approach that we have followed is by a random process of reduction of the conventional generators. We have done a large sampling of cases, 256 for each case considered and we choose the selected set of generators that cause the minimal risk. We have consider several reductions for each network The results are given in Fig. 12a for the 200 node network and Fig. 12b for the 400 node network.

These results are similar to the ones shown in Fig. 11. Despite the differences in the reduction methods and iterations schemes that are different for each case, the conventional generators that have been closed down are the same for the cases studied. The optimization can still be improved, but it is difficult to go beyond those limits in the contribution of the wind power without increasing the normalized risk above 1 . Also one can play with the main variables of the system to decide what final configuration is the best for the needs of the moment and the costs of the system. These type of choices go beyond this work.

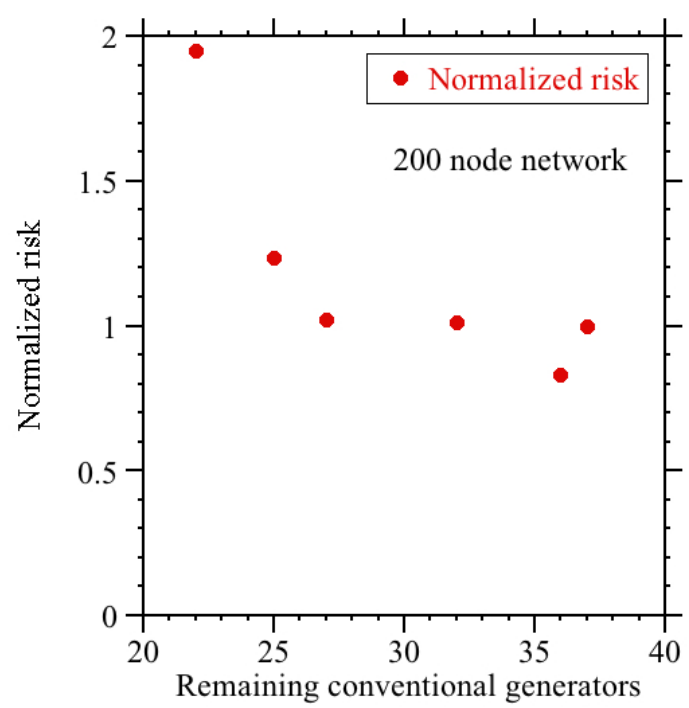

Fig. 12a. Reduction on the number of conventional generators using a random selection approach for the $\mathbf{2 0 0}$ node networks.

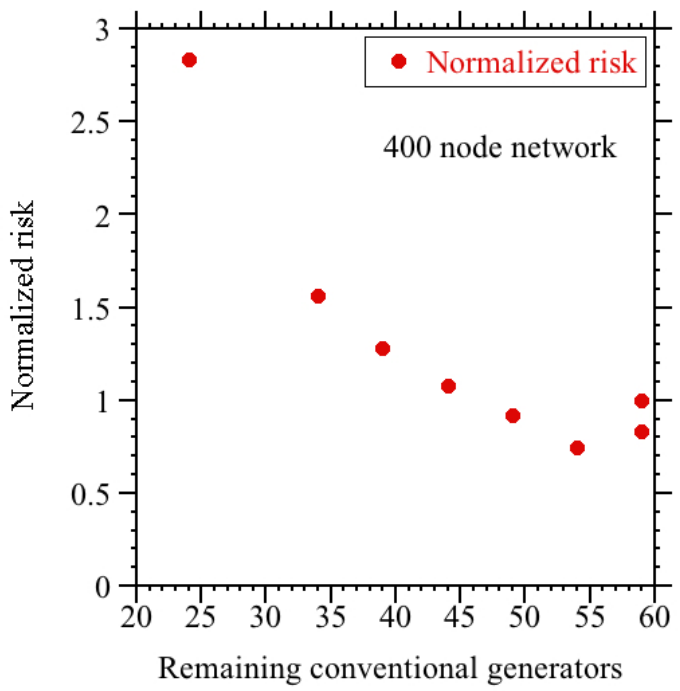

Fig. 12b. Reduction on the number of conventional generators using a random selection approach for the $\mathbf{4 0 0}$ node networks.

\section{Conclusions}

In this paper we have shown a path to optimizing power transmission systems with a significant wind penetration supported by local storage. For this work is was done on artificial transmission networks with placement of wind generation chosen and co-located storage. However the same procedure can be applied to real grids with the wind generation properly places and the variability regions chosen by real correlations in the wind. The same approach can be used for other renewable power sources with variability such as solar or both solar and wind. In addition to making the power transmission grids more realistic, additional details of the storage location and response, wind generation and dispatch can be added in future work. One of the observations in this study is that the location of the new plants can be important for the reliability of the system. In planning ahead for the incorporation of new generators in a grid, it would be useful, if not critical to study the optimization of location for the new generators under the corresponding geographical constraints.

The steps that we use in the optimization process are 1) Calculate the optimal power output for each wind plant and optimal storage based on local wind data. 2) Choose best network connections for the wind plants. 3) Optimize the penetration fraction based on the previously chosen configuration (with the medium and long time variability taken into account). Finally, 
4) Optimize the number of conventional power plants in the system. The limiting factors in the system reliability are the distribution of the generation and its variability. As the renewable generation varies, forcing the conventional generation to pick up the slack, the power flow is forced to shift, adding stress to the grid increasing the probability of failure. This is worse for the high frequency variability [10] (daily) but is still true for the slower seasonal variability. To address the shorter time scale we have developed a method for calculating both the needed storage to maintain a given local power supply. though this is not likely to be useful for the seasonal variations unless they are significantly smaller then the daily variability.

\section{References}

[1] Marco Liserre, Thilo Sauter, and John Y. Hung, "Future Energy Systems" IEEE Industrial Electronics Magazine, 10, 1932 (2010).

[2] J. M. Carrasco, L. G. Franquelo, J.T.Bialasiewicz, E. Galvan, Member, R. Portillo, M. M. Prats, J. I. León and N. Moreno, "Power Electronic Systems for the Grid Integration of Renewable Energy Sources: a Survey" IEEE Transactions on Industrial Electronics, 53, 1002-1016 (2006).
[3] Sercan Teleke, Mesut E. Baran, Subhashish Bhattacharya, and Alex Q. Huang, "Rule-Based Control of Battery Energy Storage for Dispatching Intermittent Renewable Sources" IEEE Transactions on Sustainable Energy, 1, 117 (2010).

[4] B.A. Carreras, V.E. Lynch, I. Dobson, D.E. Newman, Complex dynamics of blackouts in power transmission systems, Chaos, vol. 14, no. 3, Sept. 2004, pp. 643-652.

[5] I. Dobson, B.A. Carreras, V.E. Lynch, D.E. Newman, Complex systems analysis of series of blackouts: cascad- ing failure, critical points, and self-organization, Chaos, vol. 17, no. 2, 026103 June 2007.

[6] S. Mei, X. Zhang, M. Cao, Power Grid Complexity, Tsinghua Univ. Press, Beijing and Springer, Berlin 2011.

[7] Wind data NREL - https://www.nrel.gov/grid/windtoolkit.html

[8] Z. Wang, A. Scaglione, and R. J. Thomas, "Generating statistically correct random topologies for testing smart grid communication and control networks," IEEE Trans. Smart Grid 1(1), 28-39 (2010).

[9] B. A. Carreras, D. E. Newman, Ian Dobson, "Does size matter?" Chaos 24, 023104 (2014)

[10] D.E. Newman, B.A. Carreras, M. Kirchner, I. Dobson, The Impact of Distributed Generation on Power Transmission Grid Dynamics, in IEEE Explore, Forty-fourth Hawaii International Conference on System Sciences, Hawaii, January 2011 